\title{
Research on the Application of Phase Change Materials in the Field of Building Energy Efficiency
}

\author{
Wang Yongli ${ }^{1, a}$, Yi Xiuhong ${ }^{2, b}$ \\ ${ }^{1}$ Jilin Railway Technology College, Jilin, Jilin Province, China,132001 \\ ${ }^{2}$ Jilin Railway Technology College, Jilin, Jilin Province, China,132001
}

Keywords: Phase Change Materials, Building Energy Efficiency, Application

\begin{abstract}
Phase change materials and traditional building materials can be combined into new building materials which have regenerative and thermostat function, it has a large energy density and heat absorbing approximately constant temperature and it can effectively maintain comfortable indoor environment and reduce building energy consumption and the cost of heating and cooling required. Taking the current building energy efficiency for consideration, this paper notes that the phase change energy storage technology is an important measure to achieve energy efficiency in buildings. On this basis, we summed up the classification, characterization and preparation of phase change materials and analysis the applications and problems of phase change materials in the field of building energy efficiency.
\end{abstract}

\section{Introduction}

Along with the promotion of energy efficiency in buildings and the built environment people comfort requirements, intelligent, ecological construction materials has become an inevitable trend of development. EEB is a systematic project, saving roughly includes the following subsystems: (1) building envelope system; (2) heating and cooling air conditioning and ventilation systems; (3) solar energy and other renewable (clean) energy systems; (4) green lighting and appliances system; (5) inspection monitoring and saving recognized identification system; (6) technical advisory services system. Phase change energy storage technology is an important measure of the current building energy-saving technology, phase change materials and traditional building materials can be made of new composite materials having a thermal storage and temperature control functions. Phase Change Materials having a large energy density as well as the advantages of absorbing approximately constant temperature heat, etc., and can ease the energy supply and demand mismatch in the building on time and intensity of conflicts, reduce the negative impact of building energy consumption on the environment and the economy [1].

\section{The Classification of Phase Change Materials}

(1) According to the characteristics of energy storage, heat storage material and can be divided into cold storage material.

(2) By way of storage, divided into sensible heat storage, latent heat storage and chemical reactions storage three categories.

(3) The phase transition temperature range and can be divided into high, medium and low temperature storage material.

(4) According to the chemical composition, it can be divided into inorganic phase change materials, organic phase change materials and composite phase change materials into three categories.

(5) By way of a phase change, it can be divided into solid - solid phase transitions, solid - liquid phase change and liquid - vapor phase transition and solid - vapor phase change four categories.

\section{The Commonly Used Characterization Methods of Phase Change Materials}

(1) Differential scanning calorimetry (DSC). It can be used to measure the phase change 
temperature range and energy density storage materials, as well as providing heat of fusion, heat curing and other data.

(2) TG analysis. Phase change materials can be seen in the volatile situation and the exothermic heat storage capacity within different temperature ranges.

(3) The time - temperature curve. The method can simultaneously measure the phase change material latent heat of the phase transition temperature and other physical properties of the material by measuring the change of time exactly calculate the thermal conductivity.

(4) Scanning electron microscopy (SEM). Be prepared for a phase change materials section was observed to determine the homogeneity and stability of its structure.

(5) Fourier transformation infrared spectroscopy (FTIR). According to appearing on the corresponding wave number absorption peaks shown in the graph on the infrared spectra can be analyzed whether the product described microcapsule wall material contains a substance and the core material.

(6) Laser particle size distribution analyzer. It can automatically online measure the particle size distribution of the particle size, the average particle diameter, a relatively high resolution.

(7) X-ray diffraction (XRD). It can analyze a crystal structure of the core material, to obtain the surface material $(10 \mathrm{~nm})$ of the constituent elements and chemical bonding state and other information.

\section{The Preparation and Storage of Phase Change Energy Material}

Direct Addition. Directly to the phase change materials blended into conventional building materials, and then prepared according to conventional mixing required components and molding process has the advantage of easy to control the amount, make PCMs evenly dispersed, but there is also the phase change material easily leaks and other issues . Ziming Wang and other studies have found by direct mixing phase change material can significantly improve the hydration process, but on the compressive strength of cement paste fluidity and concrete will have some negative impact.

Direct Immersion. With melted phase change materials impregnated porous building materials such PCMs infiltrated into the porous building materials matrix. Victor Fung will wait for direct immersion method combines the PCM and the wall material, phase change after 300 cycles, the PCM wallboard phase transition temperature, latent heat value unchanged [2].

Carrier Package Method. The carrier matrix made of microcapsules or three-dimensional network structure, and then the phase change materials adsorbed and fixed therein. Takeshi K, etc. will PCMs pressed into the cross-linked polyethylene, made of micro energy capsule, then put the microcapsules added gypsum board. The main advantage of this method is no need in containers, can be directly processed molding, cooling phenomenon does not occur, the heat storage rate and high thermal conductivity.

Melt Blending Method. Phase change compatible material and substrate, the components are mixed together into a homogeneous storage material is melted. Inaba $\mathrm{H}$ and other by melt blending successfully prepared paraffin / high density polyethylene stable phase change materials.

Mixing Sintering. The prepared micron substrate material and phase change materials were uniformly mixed, then mix plus a portion of the additive milled and pressed sintered molding, resulting in energy storage materials. Zhang snow by powder sintering process will $\mathrm{Na} 2 \mathrm{CO} 3$ and Mgo composite, made from a new type of high temperature inorganic / ceramic matrix composite phase change thermal storage materials.

Porous Adsorption. Porous medium as a phase change material composite packaging materials can have the advantage of the structure integration, and because many of porous materials are tiny particles, effectively increasing the heat transfer area, improve the phase change heat transfer efficiency. In binary complex as phase change materials, the choice of lightweight ceramic adsorption of the phase change materials, and with them a variety of materials processing, surface-mount ceramic made of phase change energy storage.

Microencapsulation Method. Microencapsulated phase change is the use of a polymer as wall material, to the vicinity of the phase transition point temperature of the phase change material as the 
core material. In designing a microcapsule, you must select the appropriate method of microencapsulation of core material characteristics and performance requirements needed microcapsules. Cho and other prepared polyurethane microcapsule wall material. Shang and other phase change paraffin as a core material, cheap and polyurethane as wall material, prepared by interfacial polymerization of single and double-walled timber microencapsulated phase change materials. Under the same conditions, the double-walled timber microcapsules during the synthesis reaction fully, higher yield, better temperature stability.

Auxiliary Substances. Preparation of phase change materials, the need to add different modified materials to meet their functional requirements [3]:

(1) Nucleating agents and thickeners. Alvarado et al proposed the addition of the phase change materials homologous nucleating agent can effectively reduce the degree of supercooling;

(2) Dispersant;

(3) Thermally conductive filler. Paraffin as phase change materials for energy storage systems, in which the addition of aluminum powder to improve thermal conductivity, the study showed, after adding aluminum powder storage time can be shortened $60 \%$ and useful heat obtained significantly increase;

(4) Retardant chemicals.

\section{The Application of Phase Change Energy Material in the Field of Building Energy Efficiency}

Phase change material as a highly efficient thermal energy storage medium, in the field of building energy efficiency has an important value, with significant economic, environmental and social benefits [4].

Inject PCMs to the Wall. Since the phase change wallboard phase change thermal storage properties of building materials, so that heat transfer is greatly reduced by the retaining structure, can significantly improve the thermal comfort of the indoor environment.

Neeper studied the three main parameters affecting the plasterboard phase transition: PCMs melt temperatures, melting occurred in the temperature range, the thermal storage performance per unit area of the wallboard. Athienitis and other energy storage phase change plasterboard in a passive solar house inside the wall, and the numerical simulation and experimental study on thermal performance. The results showed that the use of phase change wallboard can reduce indoor maximum temperature $4{ }^{\circ} \mathrm{C}$, phase change materials solidification release heat equivalent of $15 \%$ of the total heating load. Wang Qidong, respectively, butyl stearate paraffin phase transition phase change materials, polyvinyl alcohol as a dispersing agent, gypsum board as the carrier prepared by directly into phase change plasterboard. The results showed that the phase change energy storage plasterboard its storage capacity is 10 times that of ordinary gypsum board, excellent durability and water absorption is $1 / 3$ of ordinary gypsum board and facilitate the use of high-humidity environment.

Inject PCMs to the Concrete Block. Phase change materials in favor of concrete hydration heat control, can effectively control the early cracks in the concrete heat, thereby improving water resistance and durability of concrete. Hawes and other studies of different types of concrete blocks in the thermal storage properties of multiple PCMs, PCMs improved by combination of technology can make a concrete block containing PCMs for heat storage capacity increased by 2 times. Zhang Dong and other studies Preparation "two-step" phase change energy storage concrete: First phase change aggregate production, aggregate and then using phase change energy storage technology using ordinary concrete preparation phase change formulated concrete. Chen Mei Zhu and other studies of the phase change materials implanted in concrete, absorbing and releasing heat of phase change materials phase transition of the properties developed a hydration heat-controlled smart concrete. Gui waves were high with half-adiabatic temperature measuring device were studied under different PCMs landfill, cement paste, mortar, concrete internal temperature changes, and discusses the impact of pre-landfill PCMs concrete internal temperature trends and control effect.

Use PCMs for Floor Heating. Heating the indoor floor heating is a relatively new way of 
building heating, it provides greater heating area, indoor levels of temperature distribution and vertical temperature gradient is small [5].

Farid and other micro-capsule technology encapsulated phase change materials do CaCL2-6H2O preparation phase change storage floor, the floor surface can effectively reduce the temperature fluctuations, thermal comfort is better. Ye Hong and other studies using a composite material phase change stereotypes radiant floor heating system as energy storage medium, the results showed the use of a melting point of about $32{ }^{\circ} \mathrm{C}$ stereotypes PCMs, indoor temperature fluctuation is very small, can greatly simplify the control system. Qiu Lin, on the one filled with salt hydrate phase change materials building ground floor storage process TH29 structure of the numerical simulation study, the simulation results of the floor structure optimal design with the measured results were compared and found that both a high degree of agreement.

Use PCMs for Air Conditioning Systems. In the air conditioning system in the application phase change energy storage technology, can effectively solve the problem of energy supply and demand does not match the time, it is an important way to achieve grid "peak load shifting Valley". Phase change materials have greater energy density, is 5 to 14 times the same volume sensible heat storage material can be directly carried out using conventional refrigeration storage unit, increase the evaporation temperature and COP values refrigeration units, thus improving energy utilization system rate, the use of phase change materials $5-10{ }^{\circ} \mathrm{C}$ in the most appropriate storage in air conditioning systems. Li Xiaoyan, etc. for the new economy PCMs storage air conditioning system were studied to establish the economic analysis model PCMs storage air conditioning system, the results show that early PCMs storage air conditioning system higher investment, but run the lowest cost, static short payback period.

Frederic Kuznik research PCMs internal partition wall used as a lightweight thermal behavior, use CODYMUR software to optimize PCM wallboard through numerical analysis, the results showed that there is an optimal PCMs thickness. Manuel Ibanez et al. [33] proposed a method of using TRNSYS software simulation, verified by concrete blocks containing PCMs established a room model, the results show that when selecting a suitable equivalent heat transfer coefficient, the method It is applicable, in addition to the storage capacity of the concrete blocks drawn and best phase transition temperature. Jo Darkwa composite phase change of one kind of concrete piping systems numerical analysis, analysis showed that the PCMs concrete duct system potential to reduce building energy consumption of air conditioning systems.

\section{Conclusions}

There are a lot of scientific researches on the combination of phase change materials and building energy-saving, but the application and promotion of phase change building materials in the market are not much. With the the growing importance of environmental protection and green building, the phase change materials will have broad application prospects in the field of ecological construction. For this aspect we can learn from foreign mature energy-saving evaluation, proposed energy-efficient building evaluation criteria for China's actual situation and gave simple and feasible analysis and calculation method.

\section{References}

[1] Chunhua Song: Building Decoration Materials World, Vol. 4 (2006) No 53, p.46-55

[2] Zhonghua Chen: Materials Review, Vol. 12 (2005) No 27, p.74-76

[3] Ziming Wang: Concrete commodity, Vol. 3 (2007) No 33, p.16-18

[4] Guohui Feng: Renewable Energy, Vol. 4 (2005) No12, p.35-37

[5] Jianli Shang: Materials Review, Vol. 3 (2010) No24, p.91-94 